\title{
MRI: a tool for measuring turbulent intensities in flow systems
}

\author{
O. Adegbite ${ }^{1}$, L. Kadem ${ }^{2}$ \& B. Newling ${ }^{1}$ \\ ${ }^{I}$ Department of Physics, University of New Brunswick, \\ Fredericton, Canada \\ ${ }^{2}$ Laboratory of Cardiovascular Fluid dynamics, \\ Department of Mechanical and Industrial Engineering, \\ Concordia University, Montreal, Canada
}

\begin{abstract}
Understanding the fluid-structure interaction and fluid dynamics downstream of an obstruction is crucial in the design and fabrication of devices that find application in both medicine and industry. It is known that the fluid flow patterns downstream of an obstruction may be very complex and are three dimensional, including the formation of vortices, recirculating flow, flow separation and the onset of turbulence. The development of any such pattern of flow might be detrimental to the optimal performance of the flow system. In this work we have used the magnetic resonance imaging (MRI) technique to investigate flow dynamics downstream of an artificial heart valve. MRI is a naturally threedimensional, non-invasive technique that finds application in clinical, biomedical research and materials research. It has the capability to visualize the internal structure of materials and also to quantify mass transport properties. In this in vitro study, we have measured the turbulent diffusivity and velocity downstream of the valve in two configurations (fully opened and partially opened). Our particular implementation of the MRI measurement (known as SPRITE imaging) is unusually robust to fast turbulent flows and has been demonstrated effective at Reynolds numbers on the order $10^{5}$, much higher than possible with most conventional, clinical MRI techniques. The results showed a low turbulent diffusivity downstream of the fully opened valve configuration, while the turbulent diffusivity is higher downstream of the partially opened valve coupled with a high-velocity fluid jet and recirculating flow. There are distinct
\end{abstract}


differences between the downstream velocity profiles of the two configurations, which have diagnostic implications.

Keywords: mechanical heart valve, aorta, spiral-SPRITE, turbulent flow, turbulence, velocity, gradient, MRI.

\section{Introduction}

Magnetic Resonance imagining (MRI) was developed more than three decades ago. It is a well established method in medicine for the imaging of brain, spinal cord, bone and other organs in the body. MRI has several unique advantages. It is both non-invasive and non destructive, which makes it a preferred choice for the investigation of physiological problems and for clinical diagnosis. MRI has further been extended to study the flow dynamics of fluid that are of interest to clinicians and non-clinicians alike. Several studies have demonstrated the use of MRI for measuring fluid velocities and visualizing flow dynamic without disrupting the target [2]. Flow is a natural phenomenon, which can exist in different forms. It can be laminar in which case the flow is orderly and steady, or it can be turbulent, characterized by a complex flow pattern. Turbulent flow is described as irregular eddying motion in which velocity and pressure perturbations occur about their mean values. These perturbations can be irregular, random and even chaotic, in both time and space. This type of turbulent flow is advantageous in mixing. In cardiovascular system however, it may cause physiological complications. In engineering facilities, it is responsible for; among other things, erosion. This complex pattern of fluid flow is often related to the interaction of fluid with the structure through which it flows or interaction of the structure with the fluid in which it is immersed. Some other physical phenomena associated with such interaction are vortex formation, flow separation, flow recirculation, etc.

Understanding the dynamics of this flow and fluid structure interaction plays a crucial role in design and fabrication of devices that are prone to these physical phenomena. An example of such is the prosthetic heart valve. This valve is widely used to replace the diseased heart valves. The most popular and frequently implanted among this family of valves is the mechanical heart valve because of its durability. However, it suffers a major drawback due to its vulnerability to blood coagulation resulting in valve related complications such as haemolysis, platelet activation and thromboembolic events [3]. This shortcoming has been attributed to the complex flow pattern and turbulent flow that characterizes the flow downstream of this artificial heart valve [4]. The first prosthetic heart valve implant of this valve was preformed in 1960, and ever since, attention has been drawn to the study of flow dynamics past such valves and to the investigation of related physiological complications. Among the methods used to study are pulse Doppler ultrasound, laser Doppler anemometry, hot wire-film anemometry, and numerical methods such as computational fluid dynamics [CFD] [3, 5]. Recently, particle image velocimetry (PIV) [6] and particle tracking velocimetry [7] were used in in vitro studies of flow dynamics downstream of prosthetic valves. These optical techniques are known for their 
high speed, measurement of instantaneous velocities. They have been used to provide information about the spatial and temporal dependence of fluid velocities under a wide range of conditions including laminar flow and turbulence. However, optical techniques are generally ineffective with opaque materials, and are often invasive. Even with some of the ultrasound-based velocimetry techniques that overcome the problem of optical opacity, there remain technical difficulties in resolving fluid within structures, which prevent access to the volume of interest [8]. MRI circumvents these problems; since it is capable of probing internal structure in any radiofrequency (RF) transparent material.

Magnetic Resonance technique performed well in quantifying the properties of steady laminar flow or flow in which the motion of the individual fluid elements were well defined. However, characterization of turbulent flows is more complex. One major problem associated with turbulent flow when using magnetic resonance is signal loss. This occurs because of the additional phase variation that is generated by the presence of turbulent fluctuation of the velocities resulting in a loss of coherence and subsequently signals attenuation. To address this challenge, methods have been developed $[9,10]$ which are capable of resolving the velocity of fast moving fluid with Reynolds number up to 10000. A Reynolds number is a dimensionless quantity which indicates whether the flow of fluid is steady (laminar) or steady on average but with small unsteady changes (turbulent). Single Point Ramped Imaging with $T_{1}$ Enhancement (SPRITE) [11] with motion encoding [1] is capable of resolving turbulent flow with Reynolds numbers of up to $10^{5}$ without signal loss. SPRITE with motion encoding is particularly well suited to characterizing highly turbulent fluid flow (in a wind tunnel style experiment) and fluid with short lived MR signals.

The SPRITE imaging sequence is a pure phase encoding method with no signal loss over a wide range of fluid velocities. This is achieved by the acquisition of a single k-space point (see theory below) at a fixed time interval after radio frequency excitation, in the presence of a constant magnetic field gradient, which is then ramped to a new value before acquisition of the next kspace point. A bipolar gradient is superimposed on the ramped gradient, which introduces sensitivity to spin motion. This approach avoids problems arising from susceptibility variations and field inhomogeneities [11]. This technique is employed in this work to investigate the complex flow pattern downstream resulting from the interaction of the bi-leaflets of a mechanical heart valve with the flowing fluid, with the aim (i) of determining velocity distribution profile downstream, (ii) visualizing the turbulent flow and (iii) estimating eddy diffusivity without signal loss or cancellation.

\section{Theory}

Magnetic Resonance Imaging relies on the fundamental properties of molecular nuclei, which interact with an externally applied static magnetic field, to obtain spatially resolved information about the structure or dynamical properties of the 
material of interest. The magnetic moment of the nucleus arises because it has spin and hence spin angular momentum. When hydrogen nuclei experience a static magnetic field, the spins re-orient nearly parallel and anti-parallel (lower and higher energies respectively) to the magnetic field and undergo a rotation about the magnetic field called precession with a frequency that depends on the static magnetic field strength.

In Magnetic Resonance, an oscillating radio-frequency (RF) pulse with a frequency equal to the precessing frequency is applied perpendicular to the static field. The absorbed energy induces transitions between the parallel and antiparallel states. The return to thermal equilibrium is referred to as relaxation. Immediately following the RF pulse, the MR signal known as the free-induction decay (FID), is acquired. The signals persist as long as the precessing nuclei remain coherent, or precess "in phase," and induce an emf in the detector coil. Over time increasing incoherence results in the decay of the signal. In MRI, a magnetic field gradient is applied across the sample, leading to a spatially variant distribution of resonant frequencies. A two dimensional Fourier transform reconstructs the image in the spatial domain. The reconstructed image can be based on the magnitude of the signal (i.e. magnitude image) or the phase of the signal (i.e. phase image).

Most conventional MRI methods acquire many data points in the presence of a constant magnetic field gradient, an approach referred to as frequency encoding. In contrast, the SPRITE method, is a purely phase encode technique that acquires a single data point at a constant time interval, $t=t_{p}$ (encoding time) after the RF pulse, and at a specific gradient value $\vec{G}$, which is incremented after each excitation. The phase encoded signal arising from a single point acquired in the presence of a magnetic field gradient, say $\vec{G}=G_{x} \hat{x}$ is given by

$$
S\left(k_{x}\right)=\int \rho(x) \exp \left(-i 2 \pi k_{x} x\right) d x
$$

where

$$
k_{x}=\frac{1}{2 \pi} \gamma G_{x} t_{p}
$$

$\gamma$ is the gyromagnetic ratio for hydrogen nuclei, $G_{x}$ is the phase encoding magnetic field gradient, $t_{p}$ is the encoding time and $\rho$ is the nuclear spin density. If the spins are in motion along this direction, they will develop a phase offset $\Delta \varphi$ that is proportional to the magnitude and duration of the gradient field given by:

$$
\Delta \varphi=\gamma \int G_{x} x(t) d t
$$

Expanding the equation with Taylor series expansion, we have

$$
x(t)=x_{o}+v t+\frac{1}{2} a t^{2} \ldots \ldots \ldots
$$

Substituting equation (4) into (3)

$$
\Delta \varphi=\gamma \int G_{x}\left(x_{o}+v t+\frac{1}{2} a t^{2} \ldots \ldots \ldots\right) d t
$$


where $x_{o}$ is the initial position encoded by the zeroth gradient moment with respect to time. Velocity $(v)$ is encoded by the first gradient moment and acceleration $(a)$ by the second. The power of SPRITE lies in the short encoding times possible, much less than the signal lifetimes of most materials, permitting the acquisition of density weighted images. This characteristic of SPRITE also makes it possible to image fast and turbulent fluid flow. In our implementation, the phase encode gradient is ramped through 2564 points, as shown in figure 1(inset). This phase encoding gradient is bipolar, switching from $G_{x}-g$ to $G_{x}+g$ during the time constant $t_{p}$, allowing for controlled separation of the first and second gradient moments in equation (5) through the use of different values of the motion sensitising gradient $\mathrm{g}$.

Typically, 8 values of $g$ were used to acquire 8 images of a $64 \times 64 \times 8$, 3 -dimensional array. $Z$ is the flow direction. The set of images, when analysed (with a custom IDL program called jAIMS designed by the Centre for the analysis of flowing fluids) give maps of the mean velocity and turbulent diffusivity.
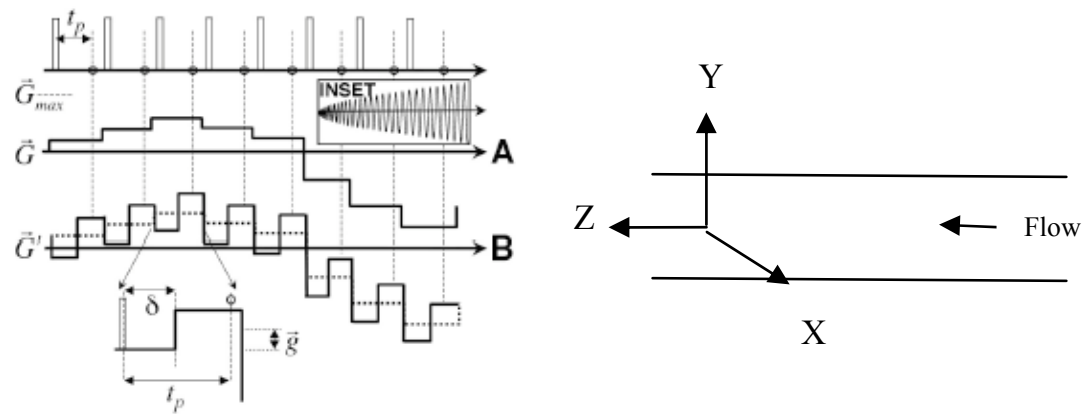

Figure 1: Shows the first few values of the magnetic field gradient for the spiral trajectory acquisition (complete trajectory in inset).

\section{Experiment}

The experimental setup for this study consists of a $1.2 \mathrm{~m}$ long glass tube with an internal diameter of $3.2 \mathrm{~cm}$. The glass tube is long enough to allow the flow to be fully developed before the fluid reaches the probe. Fitted in the middle [test section] of the glass tube in an upright position was the prosthetic aortic valve in a fully opened configuration. The working fluid is water doped with gadolinium III chloride hexahydrate for fast relaxation and the longitudinal relaxation time $\left(\mathrm{T}_{1}\right)$ is $30 \mathrm{~ms}$.

The encoding time $\left(t_{p}\right)$ is $1 \mathrm{~ms}$ and a repetition time $(T R=$ the time interval between RF pulses) is $3.2 \mathrm{~ms}$. This model was placed in a re-circulating loop that was driven by a pump with a variable voltage control $(0-100 \mathrm{~V})$, placed approximately $3 \mathrm{~m}$ away from the magnet. The water volumetric flow rate was 
13.1L/min. Spiral SPRITE was implemented on a 2.4T 32-cm i.d horizontal bore superconducting magnet. [Nolarac Cryogenic Inc. Martinez, CA]. A water cooled $7.5 \mathrm{~cm}$ i.d gradient set driven by Techron [Elkhart, IN] 8710 amplifier that generates a maximum of $50 \mathrm{G} / \mathrm{cm}$ was used for all experiments. The probe was a homebuilt 32-rung birdcage coil driven by a 2kW AMT (Brea, CA) 3445 RF amplifier. A Tecmag (Houston, TX, USA), Apollo console was employed. Image acquisitions were performed with the motion encoding gradient along the $\mathrm{z}$ direction, i.e. parallel to the bore of the magnet and the principal direction of flow.

Two set of data images were acquired; one with flow and the other without. The set of images, were analysed by jAIMS program to give the maps of the mean velocity and turbulent diffusivities.

\section{Result and discussion}

All of the results presented were for flow of Reynolds number of approximately 10000, which was calculated from $\operatorname{Re}=\frac{\rho V D}{\mu}$, for which $\rho$ is density $\left(1000 \mathrm{~kg} / \mathrm{m}^{3}\right), V$ is fluid velocity $(\mathrm{m} / \mathrm{s}), D=$ inside upstream glass tube diameter and $\mu=$ viscosity $(0.001 \mathrm{~kg} / \mathrm{m}-\mathrm{s})$. The $3 \mathrm{D}$ velocity map acquired using SPRITE with motion encoding was of two configurations: (i) fully opened leaflets or (ii) one partially opened leaflet with the other fully opened. Figure 2 shows the three orifices of the prosthetic heart valve in the fully open position. Figure 3(a), is the velocity map showing the flow pattern upstream and downstream in the fully opened configuration. The blank region in-between upstream and downstream is the location of the artificial valve. The prosthetic valve appeared blank because the valve is not transparent to the RF pulse because it is made up of pyrolitic carbon. Hence, the valve cannot be "seen" through and the content inside it cannot be visualized. However, of interest to us the flow pattern downstream of

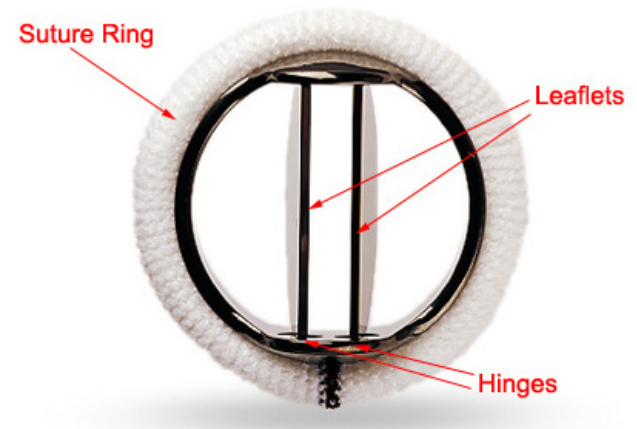

Figure 2: Depicts the prosthetic heart valve in the laboratory in a fully opened position [12]. 
this valve. The map showed a $2 \mathrm{D} Z \mathrm{ZX}$ section through a $3 \mathrm{D}(12 \mathrm{~cm} \times 5 \mathrm{~cm} \times 4 \mathrm{~cm})$ map of the z-component velocity. The $12 \mathrm{~cm}$ is from left to right but top to bottom is $5 \mathrm{~cm}$, so that the tube appears stretched in this direction. The nominal spatial resolution were $\delta z=1.875 \mathrm{~mm}, \delta x=0.781 \mathrm{~mm}$ and $\delta y=5 \mathrm{~mm}$. The actual spatial resolution is usually less because of blurring. The edges of the map are the region outside the homogenous field of the magnet. The velocity map showed the three regions of jet velocity of value $>0.35 \mathrm{~m} / \mathrm{s}$ through the three orifices. The flow through the central core region disappeared quickly as the distance extends farther away from the valve. The flow through the other two lateral orifices appears turbulent, which gradually reduced downstream as shown in figure 4(a). The unequal intensities of the velocities jet seen in figure 3(a) were due to the variation in the opening angle of the leaflet. The leaflets were opened at an angle of $10^{\circ}$ relative to each other, which might be due to the hinge mechanism [13]. This angular variation introduced unequal resistance to the flow dynamics, which in turn results in the difference in turbulent intensities through the three orifices. Figure 3(b) shows the velocity map of the other configuration of the valve (one completely opened leaflet and half closed leaflet). To achieve this configuration, a strand of thread was tied vertically along the middle of this leaflet (restriction), which held it in position as the water flows past it; a situation that mimics stenosis (narrowing). The map revealed a high velocity jet immediately downstream, which is more turbulent at both lateral orifices, with complete disappearance of the flow through the central core region as shown in figure 3(b). The jet through the open orifice extends a distance greater than 2 Diameter downstream while the flow through other orifice was directed against the glass wall. In-between, was the region of some recirculation flow, which appeared behind the restricted section of the leaflet. This region is distinct as far as 2 diameters from the valve but shows a much smaller fluid speed than the principal flow. Some fluid speeds were zero velocities and some were of negative values as seen in figure [5].

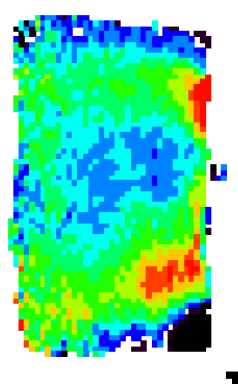

(a)

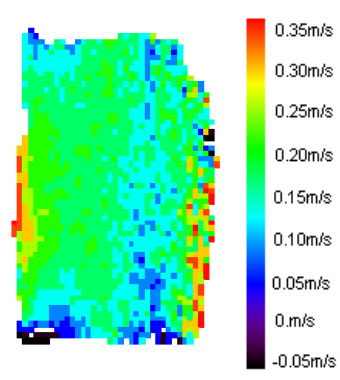

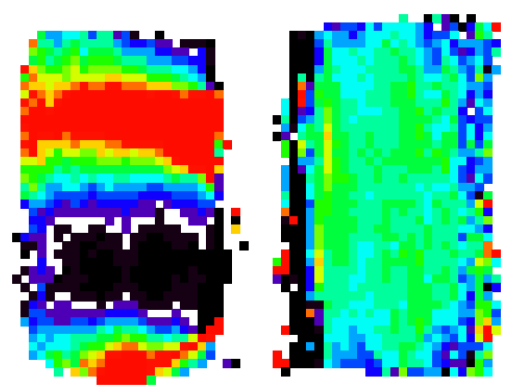

(b)

Figure 3: (a) Velocity map for the fully opened configuration, and (b) is the map for the one leaflet fully and other half closed, the white pixels indicate that the signal level fell below the noise level outside the tube. 


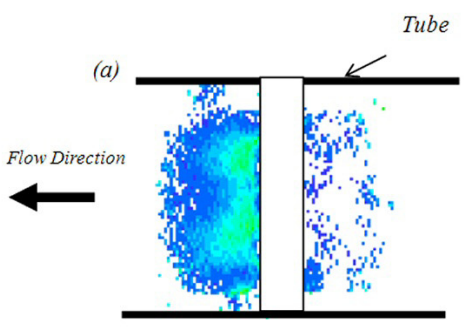

(a)

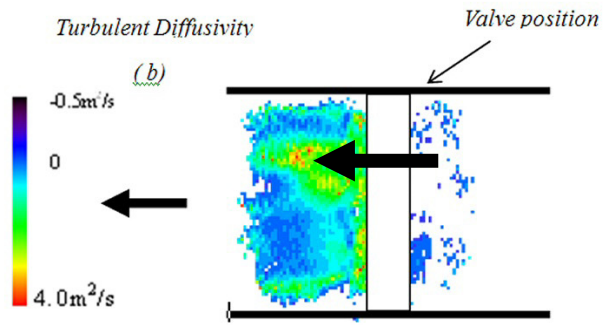

(b)

Figure 4: (a) and (b) show turbulent diffusivity maps of downstream of the heart valve when fully open (a), configuration I) and partially open (b), configuration II).

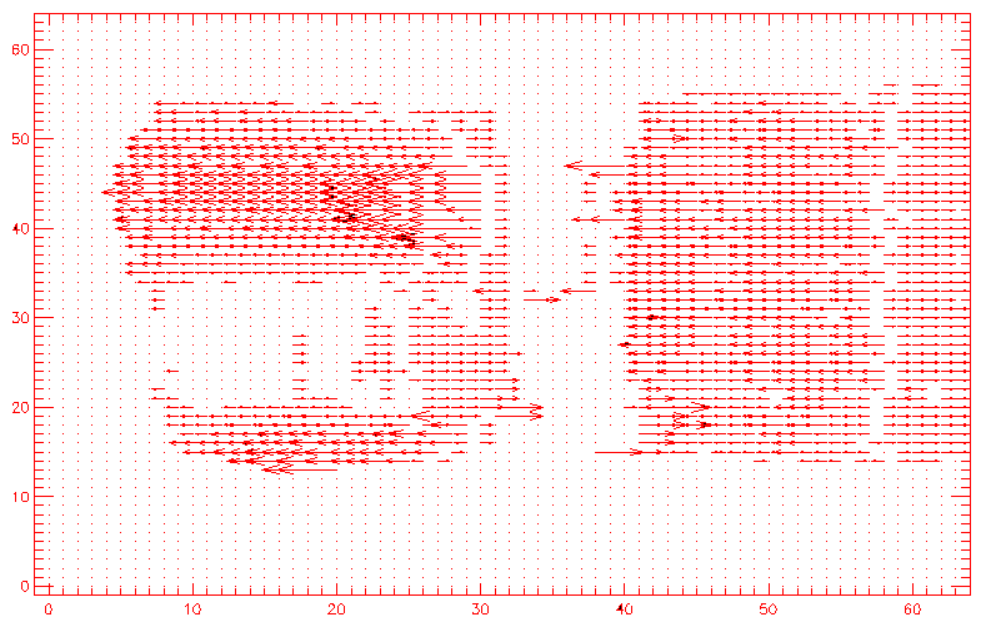

Figure 5: Showing the velocity field vector for the configuration II.

The negative velocities oppose the flow direction, some of which appeared upstream of the valve. Fluid behaviours such as this recirculation have a direct effect on cardiac function and are clinically referred to as valvular regurgitation [14].

Figure 6(a) and (b) show the velocity distribution profile for both configurations at 0.2 tube diameters downstream (notice the differing vertical scales). The jet velocities for configuration II climb up to more than $1 \mathrm{~m} / \mathrm{s}$ in the half closed leaflet and a little below $1 \mathrm{~m} / \mathrm{s}$ in the other fully opened leaflet. This is consistent with the conservation of mass principle that requires the fluid velocity to increase as it flows through the smaller cross-sectional area of the restriction. At the same time the pressure (since the fluid is incompressible) downstream is 
reduced compared to the open configuration. Immediately downstream, the flow through the central orifice completely vanished and at the two lateral orifices. Fundamentally, the relationship between the flow through a stenosed valve and its pressure difference, pressure recovery downstream and energy loss in such a flow system has been estimated [15]. We conjecture that the pressure recovery region for this configuration extended beyond that of configuration I, in conjunction with the propagation further downstream of the turbulence. In contrast, figure 4(a) showed the configuration wherein the bi-leaflets were fully opened and the jet velocities were through all the three orifices at $0.36 \mathrm{~m} / \mathrm{s}$, though the velocity through the central orifice vanishes quickly. The velocity profile at the three orifices of the valve at $0.2 \mathrm{D}$ were consistent with the profile obtained in [4], and the diffusivities reduced at some distance $>2 \mathrm{D}$ downstream. In contrast to ultrasound measurements of centreline velocity, the profiles showed the velocity distribution across the tube. Figure 4(a) and (b) above also showed the on-set and development of the turbulent diffusivities in both configurations. In configuration II, where the restriction existed, the regions of high velocities showed correspondingly higher turbulent diffusivities. The diffusivities at the half-opened bi-leaflet spread out to the wall of the tube. At the opened leaflet, the fluid jet carries turbulent velocity fluctuations further downstream than in configuration I.

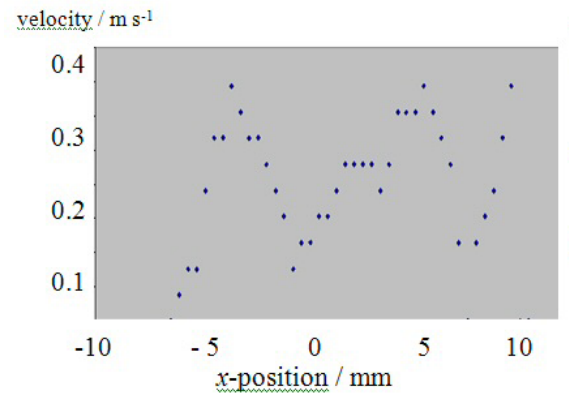

(a)

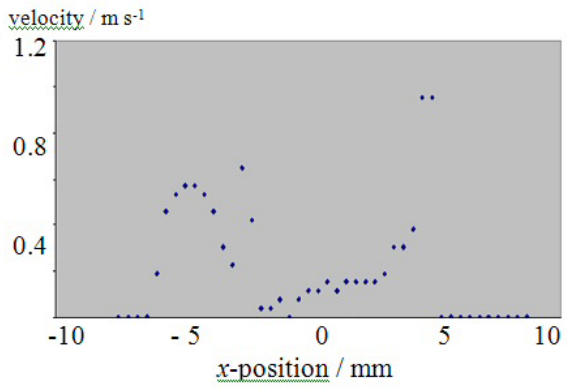

(b)

Figure 6: (a) showing the velocity distribution profile for configuration I and (b) for configuration II.

The region of lower counter flow velocities which is in-between the high turbulent diffusivities that resulted from jet velocities is bounded by regions of high shear [5]. This recirculation phenomenon has been known to have a physiological impact. For instance, it has been shown to initiate red blood cells damage or platelet activation in blood flow due to blood cells residence time in this region [12], and also, a stethoscope placed on the chest of a subject that has this flow pattern results in an audible murmuring sound, which is due to the turbulent pressure fluctuation downstream of the stenosis, and consequently results in the vibration of the aortic wall that is transmitted through the chest. In 
figure 4(a) the diffusivities of turbulent flow is very mild and disappear downstream.

\section{Conclusion}

In this study, the flow dynamics through a mechanical heart valve in two configurations of the bi-leaflets has been investigated using a specialised magnetic resonance imaging technique. The effect of the inclination of the leaflet is to create a significant velocity gradient across the flow field. The turbulent diffusivities are much higher in the second configuration, which appear to have great diagnostic implications. The MRI technique is rather naturally capable in three spatial and velocity dimensions and the efficient extension of these MRI techniques measurements with all three velocity components is underway.

\section{References}

[1] Newling B, Poirier C. C., Zhi Y, Rioux J. A., Coristine A. J, Roach D and Balcom B. J. Velocity Imaging of Highly Turbulent Gas Flow. Phys. Rev. Lett. (93), 2004

[2] Bonn D, Rodts S, Groenink M, Rafai S, Shahidzacteh-Bonn N. and Cossot P. Some Applications of Magnetic Resonance Imaging in Fluid Mechanics: Complex Flows and Complex Fluids. Annu. Rev. Fluid Mech. (40) pp 209-233, 2008

[3] Hasenkam J. M, Westphal D, Nygaard H, Reul H, Giersiepen M, and Stadkilde-Jorgensen $\mathrm{H}$. In vitro stress measurements in the vicinity of six mechanical aortic valves using Hot-film anemometry in steady flow. J. Biomechanics Vol. 21 No.3 pp 235-247, 1988

[4] Ge L, Leo H, Sotiropoulos F. and Yoganathan A. P. Flow in a mechanical Bileaflet Heart Valve at Laminar and Near-Peak Systole flow Rates: CFD simulations and Experiments. J. Biomech. Engng 127 (5), 782-797, 2005

[5] Yokoyama Y, Medart D, Hormes M, Schmitz C, Hamilton K, Kwant P B, Takatani S, Schmitz-Rode T and Steinseifer U. CFD simulation of a novel bileaflet mechanical heart valve prosthesis: an estimation of the Venturi passage formed by the leaflets. Int J Artif Organs. 29(12):1132-9, 2006

[6] Lim W. L, Chew Y. T., Chew T. C and Low H. T. Pulsatile flow studies of a porcine bioprosthetic aortic valve in vitro; PIV measurements and shear-induced blood damage. J. Biomech. 34 (11);1417-1421, 2001

[7] Balducci A, Grigioni M., Querzoli G., Romanno G. P, Daniele C., D'Avenio G., and Barbaro V. Investigation of the flow field downstream of an artificial heart valve by means of PIV and PTV. Experiments in fluids. (36) pp 204-213, 2004

[8] Song - I Han, Kimberly L. Pierce, and Pines. NMR Velocity Mapping of Gas Flow around Solid Objects. Phy. Rev. E (74) pp 0613023-5, 2006

[9] Rodriguez A. O. Study of Turbulent flow using Half-Fourier Echo-planar imaging. Brazilian Journal of Physics V(36) (1) pp 1-3, 2006 
[10] Guilfoyle D.N, Gibbs P, Ordidge R. J. and Mansfield P. Real-Time Flow Measurement using echo-planar imaging. Magnetic Resonance in Medicine (18) pp 1-8, 1991

[11] Balcom B. J, MacGregor R. P, Beyea S. D, Green D. P, Armstrong R. L, and Bremmer T. W.. Single-Point Ramped Imaging with T1 Enhancement (SPRITE), J. Magn. Reson. A (123) pp 131-134, 1996

[12] Govindarajn V, Udaykumar H.S. and Chandran K. B. Two Dimensional simulations of flow and platelet dynamics in the hinge region of a mechanical heart valve. J Biomech Eng. 131(3), 031002, 2009

[13] Detullio M. D, Cristallo K, Balaras E. and Verzicco R. Direct numerical simulation of the pulsatile flow through an aortic bi-leaflet mechanical heart valve. J Fluid mech. V(622) pp 259-290, 2009

[14] Chatzimavroudis G. P. Blood flow measurement with magnetics resonance phase velocity mapping. Measurement (37) pp 201-212, 2005

[15] Clark C. Energy losses in flow through stenosed valves. J. Biomechanics. (12) pp 737-746, 1979 